\title{
บUisuersily
}

\section{LSTM Classification of sEMG Signals For Individual Finger Movements Using Low Cost Wearable Sensor}

Millar, C., Siddique, N., \& Kerr, E. (2020). LSTM Classification of sEMG Signals For Individual Finger

Movements Using Low Cost Wearable Sensor. In 2020 International Symposium on Community-Centric Systems, CcS 2020 (pp. 1-8). [9231515] (2020 International Symposium on Community-Centric Systems, CcS 2020). IEEE. https://doi.org/10.1109/CcS49175.2020.9231515

Link to publication record in Ulster University Research Portal

Published in:

2020 International Symposium on Community-Centric Systems, CcS 2020

Publication Status:

Published (in print/issue): 20/10/2020

DOI:

10.1109/CcS49175.2020.9231515

\section{Document Version}

Publisher's PDF, also known as Version of record

\section{General rights}

Copyright for the publications made accessible via Ulster University's Research Portal is retained by the author(s) and / or other copyright owners and it is a condition of accessing these publications that users recognise and abide by the legal requirements associated with these rights.

\section{Take down policy}

The Research Portal is Ulster University's institutional repository that provides access to Ulster's research outputs. Every effort has been made to ensure that content in the Research Portal does not infringe any person's rights, or applicable UK laws. If you discover content in the Research Portal that you believe breaches copyright or violates any law, please contact pure-support@ulster.ac.uk. 


\section{LSTM Classification of sEMG Signals For Individual Finger Movements Using Low Cost Wearable Sensor}

\author{
Christopher Millar \\ Faculty of Computing, Engineering and Built \\ Environment \\ Ulster University \\ Derry, N. Ireland \\ millar-C21@ulster.ac.uk
}

\author{
Dr. Nazmul Siddique \\ Faculty of Computing, Engineering and Built \\ Environment \\ Ulster University \\ Derry, N. Ireland \\ nh.siddique@ulster.ac.uk
}

\author{
Dr Emmett Kerr \\ Faculty of Computing, Engineering and Built \\ Environment \\ Ulster University \\ Derry, N. Ireland \\ ep.kerr@ulster.ac.uk
}

The electrical activity of the muscles that control finger movements can be extracted during the performance of these movements and using machine learning techniques, the myoelectric signals can be decoded and classified according to the movement that generated the specific signal. The focus of this paper is to classify sEMG signal using easily accessible cheap hardware to capture the signal. Furthermore, to employ neural networks to classify the signal using established methodology i.e. feature extraction, with the highest possible accuracy. To classify these sEMG signals, an LSTM network has been developed and was able to classify 12 individual finger movements with accuracies reaching $90 \%$.

\section{Keywords-sEMG, LSTM, Myo, Finger Movement Classification}

\section{INTRODUCTION}

Endowing robots with grasping capabilities similar to the dexterity demonstrated by humans is a substantial challenge [1], yet it will provide great autonomy to robots and will allow them to interact with humans in a natural way. Robotic grasping is a highly complex problem which requires knowledge about the shape, mass distribution, and friction of the object to grasp, but is also conditioned by the purpose of the proposed task to be executed with the object. Standard approaches to robot grasping try to take all these aspects into consideration and devise a plan on how to move individual fingers to achieve a suitable grasp. A promising alternative consists on learning grasping movements and configurations from human examples. However, in order to train a robotic grasping system using human demonstrations it is necessary to know precisely how a human performs this task. This paper presents the contribution towards human demonstrations for robotic grasping by identifying different finger movement using a low-cost electromyography (EMG) system.

A popular method of examining human body movements in biomechanics is through the electrical outputs of the muscles involved in the movements being investigated, known as electromyography [2]-[5]. One technique for measuring the EMG signal is using intramuscular needles inserted into the muscles to detect the myoelectric signal. Although very accurate, this method is a highly invasive procedure that requires a medical expert to ensure the safety of the subjects and correct placement of the electrodes on the muscles [6]. Another approach is through surface electromyography (sEMG), a non-invasive method that involves placing an electrode on the surface of the skin above the muscle regions to detect the muscles electrical activity. A comparative study concluded that there is no significant difference in the accuracy of the classification, therefore sEMG is generally preferred due to its non-invasive nature [6].

The sEMG signal has been used in models that have been developed for classifying individual finger movements with a high degree of accuracy [4], [7]-[9]. Through the development of feature extraction techniques in combination with machine learning methods, improved performance of sEMG signal classification has been achieved which makes it a suitable technique for application with robotic systems [10]. Applied to robotics it can be an intuitive method of controlling or training an anthropomorphic robotic system based on real world human demonstrations.

\section{RELATED WORK}

In [2], [11] and [12] a small selection of basic movements were performed i.e. simple directional arm movements up, down, left \& right [9], elbow flexion and extension and forearm rotation [2] as well as functional gestures: power grip, precision grip, open hand, pointed index finger and wrist flexion/extension. The classification methods varied between these papers with [2] and [9] employing a back propagation neural network (BPNN), whereas [14] used an SVM classifier. A selection of commonly found time and frequency domain features were extracted from the original EMG signal in all these papers. All these works investigate similar type of movements, movements that generate larger muscle contractions, and therefore generate signals with larger signal to noise ratio. Furthermore, these papers use high sampling ratio e.g. $1000 \mathrm{~Hz}$ devices, for collecting the data. This has been shown to be beneficial when performing sEMG signal classification as they can generate more data per movement making it easier to discriminate between the movement classes [13], [14].

Modelling finger movements through decoding EMG signals generated by the muscles of the forearm when contracting to perform flexion and extension of the digits is a 
relatively new application of existing EMG based techniques. In [4] the authors used an array of 32 sEMG electrodes wrapped around the forearm of the user to collect the sEMG data of a total of 10 finger movements i.e. flexion/extension of each finger and 2 grouped movements of the middle, ring and little fingers. The detection device sampled data at a frequency of $2000 \mathrm{~Hz}$ and four commonly found time domain features were extracted from the original signal and used as inputs into a feedforward neural network (FNN). To improve the classification of the network the features were extracted from overlapping windows. In a follow up work the authors classified individual finger movements of amputee subjects using the same protocols [7]. The research presented in [9] revolved around a benchmark database: Ninapro ${ }^{1}$ where the authors collected data from able bodied and amputee subjects using different sEMG technologies whilst performing a benchmark set of movements that are associated with activities of daily living [15]. The authors collected sEMG signals using a medical grade device with ten electrodes and a sample frequency of $100 \mathrm{~Hz}$ and positioned the electrodes uniformly around the subjects forearm. A Support Vector Machine (SVM) was utilized to classify 12 different movements, ten of which were individual finger movements, i.e. extension/flexion index finger. Although the author does not appear to employ any form of signal segmentation which could account for not achieving higher classification of the signals but as they are using a detection device with a large sampling rate and investigating movements that generate large signals they are still able to produce results with high levels of accuracy [9]. Using this medical grade equipment the authors were able to successfully classify with a relatively high degree of accuracy, the authors also noted that this is a commercial device that records a rectified signal rather than raw data and that this can have an effect on the final classification accuracy. These studies yielded classification accuracies between $80-90 \%$ for individual finger movements, however, they employed expensive medical grade EMG detection systems [9] or detection systems with high sample rate [4]. Systems like these are not always a viable option for researchers due to the cost of the systems and can require a level of expertise to correctly setup and operate.

The introduction of commercially available cheap wearable sensors offers an alternative method of sEMG signal capturing. Recently the Myo gesture control armband ${ }^{2}$ has been released which has multiple applications i.e. a controller for an intuitive music device [16], sign language translation [17] and controlling robots and prosthetic limbs [2]-[4], [9], [11], [18]. However, these devices typically have a lower sampling rate (e.g. Myo gesture control armband samples at $200 \mathrm{~Hz}$ ) and a lower signal to noise ratio making the signal classification problem more difficult. The development of an intuitive system in conjunction with easily accessible wearable sensors could lead to a sophisticated grasping model that is based on real world human demonstration. Such a system will then dictate the movements performed by an anthropomorphic robotic hand when picking up objects and performing tasks associated with activities of daily living. By measuring and collecting the data of the electrical impulses with one of these commercial devices and combining it with state-of-the-art machine learning algorithms e.g. neural networks, and robust feature selection, the sampling rate drawback can be mitigated against and a high level of accuracy can still be achieved [16], [19]-[22].

Research using the Myo armband has shown how neural networks have been applied to sEMG signal classification i.e. convolutional neural networks (CNN) and Long Short Term Memory (LSTM) networks [8], [19], [23]. Stephenson et al (2018), used the sEMG signal pattern as an image input into a $\mathrm{CNN}$ and performed classification on five finger flexion movements as well as seven gestures which included four combinations of flexion movements involving the thumb and one of the other fingers i.e. index [8]. In [23], LSTM and $\mathrm{CNN}$ networks were employed as the method of signal classification and were compared against each other. A bespoke architecture that combined LSTM layers with a single CNN layer that was named LSTM-CNN (LCNN). The authors recorded their own dataset that included some basic hand and wrist gestures i.e. wrist extension \& closed fist. In [19], using an LSTM network and a mixture of different domain features i.e. Time \& Frequency, they were able to classify some basic hand gestures i.e. open hand and closed fist. Further research has been carried out in [17], [24], [25] using the Myo armband. [17], used a CNN to classify signals extracted from the subjects when performing any of 30 sign language gestures. In [24] six basic hand gestures e.g. open hand and cylindrical grasp, were classified using a set of five time domain features extracted from overlapping windows and input into an SVM classifier. The gestures involved in these research studies require coupled movements of the fingers and arms which can generate signals with greater amplitudes than that of the signals generated by individual finger movements.

As demonstrated in the majority of the papers that have been reviewed, classification of hand gestures can be achieved using a wide range of classification methods e.g. SVM, LDA, ANN, LSTM, and CNN. This can be down to the fact that the generated sEMG signal of these dynamic gestures i.e. wrist flexion or gestures involving multiple fingers, generally generate signals with more pronounced amplitudes. Whereas, with individual finger movements the detected signal will be a lot more difficult to distinguish between movement classes due to the reduced amplitudes of the already inherently weak signals that are generated and cross over where the same muscle control multiple fingers i.e. mid, ring and little fingers.

Furthermore, a range of different devices have been evaluated in the reviewed studies and it is evident that sEMG signal capture systems with higher sampling rates have demonstrated the ability to discriminate subtle individual finger movements. Whereas, devices with lower sampling rates, e.g. Myo armband, are less able to complete the same task. Another factor that has been highlighted is that when more movement

\footnotetext{
${ }^{1}$ http://ninaweb.hevs.ch/
}

${ }^{2}$ https://support.getmyo.com/hc/en-us/articles/203398347Getting-started-with-your-Myo-armband 


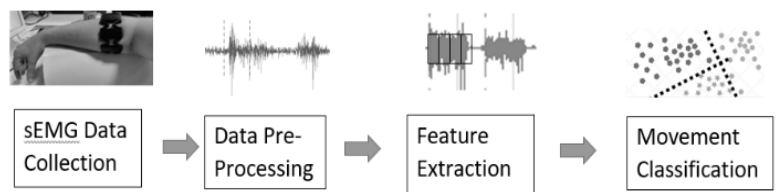

Fig. 1. Abstract view of proposed system architecture

classes are introduced for classification the final classification accuracy is often reduced. This affects the classification of all devices but more so devices with reduced sampling rates.

The focus of this paper is to develop a neural network that can classify individual finger movements from the sEMG signal detected using wearable EMG detection hardware. Fig. 1 shows an abstract view of the pipeline of the proposed system that is to be developed as part of the research being conducted.

The rest of this paper is organised as follows: Section III outlines the technology being used along with the architecture of the neural network that was created, section IV details the experimental protocols that were followed. Section V details the results of the experiments and finally a conclusion in section VI.

\section{Methodology}

Fig. 1 illustrates an abstract overview of the proposed pipeline of the system and this following section will describe each stage of the pipeline. Section A provides a detailed description of the technology being used for data collection: the Myo armband. Section B focuses on feature extraction techniques. The final section will describe the LSTM network architecture used for the classification of the movement signals.

\section{A. sEMG Device}

The Myo armband, shown in Fig. 2(a), is a wireless device that detects the inherently weak EMG signal generated by the forearm muscles in addition to other spatial information e.g. Orientation. The Myo is made up of eight medical grade stainless steel dry electrodes that detect the electrical output of the muscles found in the forearm that are responsible for the dexterous movement of the fingers and thumb i.e. Flexor Digitorum Profundus or Extensor Digitorum Communis. The Myo armband represents the EMG signal and normalises it to within a value range between -128 and 128 . These values are the amplified detected signal that is measured by the eight electrodes. The Myo armband is also equipped with a nine axis inertial measurement unit (IMU) that contains a three axis gyroscope, three axis accelerometer and a three-axis magnetometer that can measure the speed of movements and orientation of the armband in 3D space. The addition of these extra sensors allows for the potential fusion of sEMG and IMU data from a wearable device. By fusing the data provided by the multiple sensors within the Myo it could allow for more advanced autonomous control systems by giving the system more information to analyse and learn from [13], [14], [19]. The main drawback with using the Myo is the fact that it has a sample rate of $200 \mathrm{~Hz}$ which is lower than medical grade sensors that are used in other sEMG signal classification papers e.g. [7], [18], [20], [21]. However, whilst this is a valid concern the difference between the overall classification accuracy when

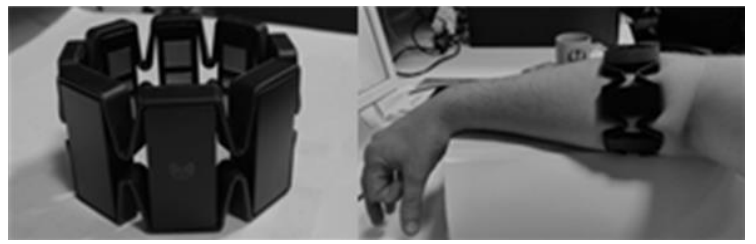

(a)

(b)

Fig. 2. (a) Myo Armband (b) Subject wearing device around forearm

using the devices with a lower sampling frequency i.e. Myo, has been demonstrated to be less than 5\% when employing techniques like feature extraction and sliding windows to reduce the impact of the low sampling rate [13], [14].

\section{B. Feature Extraction}

A large number of possible features used to reduce the dimensionality of the dataset can be found in the literature. These features can be split into three common domains: Time, Frequency and Time-Frequency. Features within these domains have all been used as a method of improving sEMG signal classification accuracy. Time domain features are the most commonly used throughout the literature as they are the most efficient in terms of calculation time and classification result. The initial set of time domain features used are the most commonly used in sEMG signal classification, these features will be tested individually and within different sets to find the features that produce the best classification performance. The features extracted from the EMG signals in this research are:

- Mean Absolute Value (MAV) is the most commonly used feature found in the literature. It is the average of the absolute value of the EMG signal for each window that the signal has been segmented into. $X$ is the MAV of the signal in segment $i$ which is $N$ samples long. $X_{k}$ is the $\mathrm{k}^{\text {th }}$ sample in segment $i$ and $I$ is the total number of segments that the original signal sample has been split into.

$$
\overline{\mathrm{x}_{1}}=\frac{1}{\mathrm{~N}} \sum_{\mathrm{k}=1}^{\mathrm{N}}\left|\mathrm{x}_{\mathrm{k}}\right| \text { for } \mathrm{i}=1, \ldots . ., \mathrm{I}
$$

- Waveform Length (WL) is another popular feature from the time domain that has been used throughout the literature. This feature is used to quantify the complexity of the waveform in each signal segment. It is the cumulative length of the waveform over the entire signal segment. $X_{n+1}-X_{n}$ is the difference in consecutive sample voltage values.

$$
\mathrm{WL}=\sum_{\mathrm{n}=1}^{\mathrm{N}-1}\left|\mathrm{x}_{\mathrm{n}+1}-\mathrm{x}_{\mathrm{n}}\right|
$$

- Variance $(V A R)$ has been used as a time domain feature in gesture recognition studies. Variance is a measure of the power of an EMG signal and represents the deviation of the EMG signal from its mean value.

$$
\operatorname{VAR}=\frac{1}{\mathrm{~N}-1} \sum_{\mathrm{n}=1}^{\mathrm{N}} \mathrm{x}_{\mathrm{n}} 2
$$


- Slope Sign Change (SSC) has been extensively used in the literature, it represents the number of times that the EMG signal changes between the positive and negative slope changes between three sequential segments. The threshold is normally selected between $50 \mu \mathrm{V}$ and $100 \mathrm{mV}$.

$$
\mathrm{SSC}=\sum_{\Pi=2}^{\mathrm{N}-1} \mathrm{f}\left(\mathrm{x}_{\mathrm{n}}-\mathrm{x}_{\mathrm{n}-1}\right) \mathrm{x}\left(\mathrm{x}_{\mathrm{n}}-\mathrm{x}_{\mathrm{n}+1}\right)
$$

- Autoregressive Modelling (AR) is used for classification of gestures and finger movements as it has been shown that the sEMG spectrum changes when muscle contractions are performed. $a_{i}$ are the AR coefficients, $N$ is the model order and $e_{k}$ is the residual white noise. Different coefficients have been found to be suitable for feature extraction of EMG signal ranging from 1 to 10 [13], [26]-[29]. In this paper the $1^{\text {st }}$ and $2^{\text {nd }}$ orders are used.

$$
\mathrm{X}_{\mathrm{k}}=\sum_{\mathrm{i}=1}^{\mathrm{N}} \mathrm{a}_{\mathrm{i}} \mathrm{X}_{\mathrm{k}-\mathrm{i}}+\mathrm{e}_{\mathrm{k}}
$$

- Zero Crossings (ZC) represents frequency information of an EMG signal but it is defined in the time domain. It is the number of times that the amplitude of the EMG signal crosses the zero amplitude level. As a way of avoiding signal fluctuations or signal noise a threshold condition is implemented.

$$
\begin{gathered}
\mathrm{ZC}=\sum_{\mathrm{n}=1}^{\mathrm{N}-1}\left[\operatorname{sgn}\left(\mathrm{x}_{\mathrm{n}^{*}} \mathrm{x}_{\mathrm{n}+1}\right) \cap\left|\mathrm{x}_{\mathrm{n}}-\mathrm{x}_{\mathrm{n}+1}\right| \geq\right. \text { threshold } \\
\operatorname{sgn}(\mathrm{x})= \begin{cases}1 & \text { if } \mathrm{x} \geq \text { threshold } \\
& 0, \text { Otherwise }\end{cases}
\end{gathered}
$$

- Willison Amplitude (WAMP) is the resulting number of times that the difference between two adjacent signal segments amplitude exceeds a pre-set threshold as a method of reducing noise similar to ZC and SSC.

$$
\begin{aligned}
& W A M P=\sum_{n=1}^{N-1} f\left(\left|X_{n}-x_{n+1}\right|\right) \\
& f(x)=\left\{\begin{array}{c}
1 \text { if } x \geq \text { threshold } \\
0, \text { Otherwise }
\end{array}\right.
\end{aligned}
$$

- $\quad$ Root Mean Square (RMS) this is the square root of a signal segment's mean value. This feature has been used in combination with other time domain features in many sEMG signal classification models.

$$
R M S=\sqrt{\frac{1}{N} \sum_{n=1}^{N} x_{n}^{2}}
$$

- $\quad$ Standard Deviation (STD) measures the total variation of a particular set of values against the mean of the population. This time domain feature has been used successfully in combination with other time domain features[11].

$$
\sigma=\sqrt{\frac{1}{N} \sum_{i=1}^{N}\left(x_{i}-\mu\right)^{2}}
$$

- Mean Absolute Deviation (MAD) is a measure of the average distance between the mean of a dataset and each data value within it. This feature was used by [30] when using the Myo as part of the authors hand pose recognition system.

$$
M A D=\frac{1}{N} \sum_{i=1}^{n}\left|x_{i}-m(X)\right|
$$

- $\quad$ Kurtosis (KURT) is used as measure of how outlier-prone a distribution of a dataset is.

$$
K u r t=\frac{\frac{1}{n} \sum_{i=1}^{n}\left(x_{i}-\bar{x}\right)^{4}}{\left(\frac{1}{n} \sum_{i=1}^{n}\left(x_{i}-\bar{x}\right)^{2}\right)^{2}}
$$

A technique that can be employed to enhance the effectiveness of feature extraction is the use of overlapping sliding windows [4], [7], [9], [31], [32]. Overlapping windows are used as a method of retaining as much information as possible when extracting features but also for reducing the dimensionality of the original signal. By using overlapping windows the amount of data that is extracted from the signal is increased which potentially increases the classification accuracy of the proposed system. Selection of the window size has been chosen based on previous works found in the literature, the most common window size found was $200 \mathrm{~ms}$ [4], [7], [9], [14], [32]. A window overlap of $20 \mathrm{~ms}$, has been selected to mitigate the reduced sample rate of the Myo device and provide as much data from the reduced original signal as possible to the LSTM classifier.

\section{Classification using LSTM Network}

LSTM is a recurrent neural network (RNN) that is trained though a gradient based learning algorithm that was introduced as a solution to the problems with error block-flow found in other "Back Propagation Through Time" (BPTT) and "RealTime Recurrent Learning (RTRL)" networks. The LSTM network forces constant error flow through the internal states of the special units found within the LSTM network architecture [33]. The introduction of multiplicative input gate units and output gate units allows for constant error flow. These gated cells control the flow of data depending on its strength and importance by either passing the contents on to the next cell or by blocking the information. This mechanism is controlled by the modification of the weights through the networks learning process [33]. Fig. 3, shows an overview of the architecture that was used to classify sEMG signals in this work.

The LSTM network that has been developed for this work is comprised of six different layers. The initial input layer is a sequence input layer that allows sequential data to be input into the network. Each movement sequence sample is made up of an input vector that represents each feature that has been extracted from the original signal of each of the eight individual sEMG 


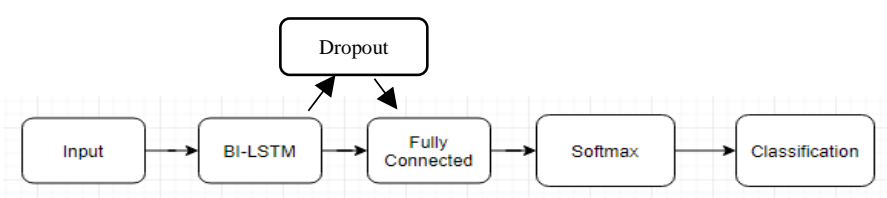

Fig. 3. LSTM Architecture

sensors. To further optimize the LSTM network the input data was normalized using the Z-score of the input [34]. This adheres to the protocol followed in [19], [23].

The sequences are input into a bidirectional LSTM (biLSTM) layer which enables the network to learn bi-directional long-term dependencies between the different time steps of the sequential data allowing the network to predict using the entire sequential input [22]. The number of hidden units in the biLSTM layer was empirically tested during the experiment to find the configuration that produced the highest classification accuracy.

A dropout layer was added after the bi-LSTM to reduce overfitting [23]. Dropout is a method of network regularization that attempts to reduce the co-adaption of the hidden units within the network. The dropout layer operates by randomly deactivating hidden units with a probability of $p$, in this case $p$ $=0.3$ [23]. The fourth layer in the network is the fully connected layer that multiplies the inputs by a weight matrix and adds a bias vector to the input, this layer then feeds into the softmax layer where a softmax function is applied to the activation. Finally there is a classification layer that computes the cross entropy loss for classification networks with mutually exclusive classes. Further parameters can be adjusted to suit the particular problem that is being investigated e.g. mini batch size, number of hidden units, additional layers.

\section{DATA ACQUISITION AND PRE-PROCESSING}

This section describes the protocols followed during the experimentation process will be outlined. The main focus will be on how the data was collected and the pre-processing methods that were applied to the EMG signal.

\section{A. Data Acquisition Protocol}

The Myo was placed securely around the widest part of the subjects forearm. The subjects left arm was placed on a small platform, $16 \mathrm{~cm}$ in height, in an effort to remove any muscle activity that would be used to hold arm in the air, as shown in Fig. 2 (b). The hand was in a relaxed position with no contact being made with the table below. Flexion and extension of the fingers and thumb along with abduction and adduction of the thumb were performed seventy times by the subject along with a rest pose. The samples were randomly split into two sets, one for training containing $728(80 \%)$ samples and a test set containing 182 (20\%) samples of each movement [17]. A script was created in Matlab ${ }^{3}$ to prompt the subject to begin the movement and when to return to rest position. An initial 4 second rest period began each trial, once the user was prompted to perform the movement the pose was held for 5 seconds and then returned to rest position to complete the data collection

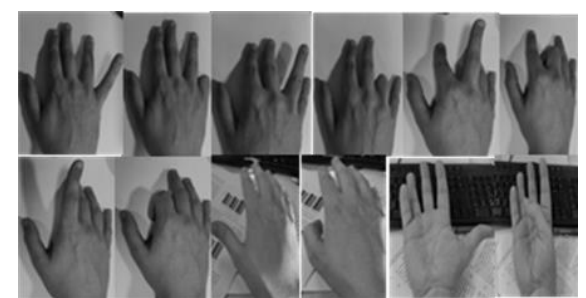

Fig. 4. Finger Poses conducted for Classification. From (Top Row ) Left to Right: Little Extension; Flexion; Ring Extension; Flexion; Mid Extension; Flexion; (Bottom Row) Index Extension/Flexion; Thumb Extension; Flexion; Thumb Abduction; Adduction

process. Using Matlab and a specifically designed toolbox, Myo SDK MATLAB MEX wrapper [35], the raw signal data was extracted from the Myo armband and imported into Matlab as the subject was performing individual finger movements i.e. Index extension/flexion, as shown in Fig. 4.

\section{B. Data Pre-Processing}

The Myo armband transmits the detected signals over Bluetooth via the Bluetooth dongle to the PC. The Myo has a built in notch filter that filters out signals at $50 \mathrm{~Hz}$, this is done to remove any interference with the EMG signal caused by European power line interference. This interference is caused by electrical interference of other systems e.g. Myo's battery, with the EMG signal. No other filtering was completed on the recorded signal. The next stage of the protocol involved removing the surplus data. As aforementioned, the movement data started after the initial 4 second rest period and ended 5 seconds later. All data before 3.5 seconds was removed, this allowed a small 0.5 second buffer to allow for user error in case of the action starting before the 4 second mark. Cropping the samples to a fixed consistent length is also important when working with LSTM networks, as with other types of RNN's, as they require the input sequence samples to be of the same length. LSTM will automatically pad all samples that are shorter than the longest sequence in the data set. The addition of too much padding can distort the signals and therefore can lead to a reduction in classification accuracy [36]. Fig. 5 shows an example of each EMG channel's signal when performing the different finger movements.

\section{RESULtS}

An initial set of experiments were conducted where a network was created using a single set of features that included all the time domain features discussed in Section III.B. The average result from 30 trained LSTM networks when classifying 13 movements (12 index/flexion movements \& 1 rest pose) along with the best single performance of a single network was recorded. Table 1 shows the parameters used for the LSTM network that will remain the same throughout the testing.

Table 1: LSTM Base Parameters

\begin{tabular}{|c|c|c|c|c|c|c|}
\hline ЕРОСН & Mini Batch & Normalization & Dropout & Weights & $\begin{array}{l}\text { No of } \\
\text { Movements }\end{array}$ & Features \\
\hline 25 & 56 & ZSCORE & 0.3 & GLOROT & 13 & ALL \\
\hline
\end{tabular}

\section{${ }^{3}$ https://uk.mathworks.com/}


To improve the classification accuracy of the network being implemented in this current research the number of hidden units in each layer were incrementally adjusted and additional biLSTM layers were added to find the best combination that produces the highest classification accuracy possible. The number of hidden units was incrementally increased until the average classification accuracy no longer increased. In the figure below, Fig. 5, it shows the average classification for each of the networks trained when incrementing the number of hidden units along with the result of the best performing network.

As shown in Fig. 5 there is a steady increase in the average accuracy that peaks when using 180 hidden units in the biLSTM layer. The average accuracy achieved here is $84.49 \%$, however, the single best performing network that uses 180 hidden units achieved an accuracy of $87.36 \%$. There were other networks that achieved a higher single accuracy e.g. $87.91 \%$, when using less hidden units, 160 or 170 therefore two layer LSTM networks using both 160 and 170 hidden units were also tested.

A second bi-LSTM layer was added to the network with a dropout layer and the hidden units for that layer were incremented again, starting at 10 hidden units, until there was no more improvement on the average classification accuracy. Fig. 6, below, shows that the when using 2 bi-LSTM layers with 160 and 30 hidden units in the first and second layers respectively, the average accuracy was increased to $87.07 \%$.

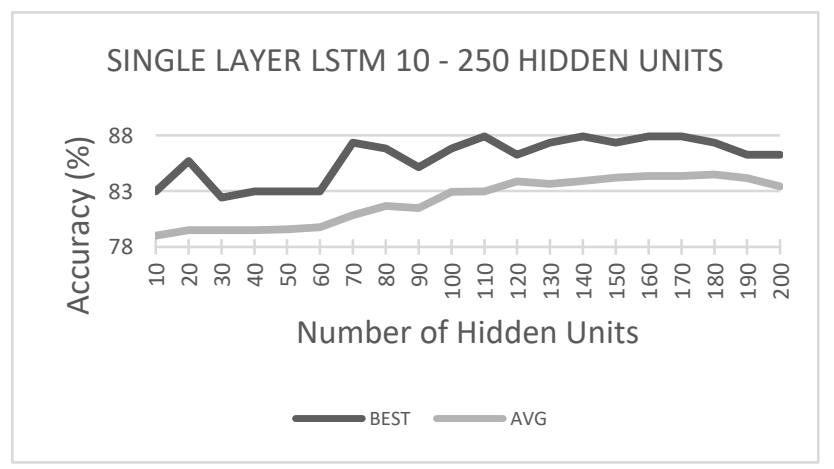

Fig. 5. Results of LSTM with single bi-LSTM layer when incrementing number of hidden units

\section{Layer LSTM 160 - 180 Hidden Units}

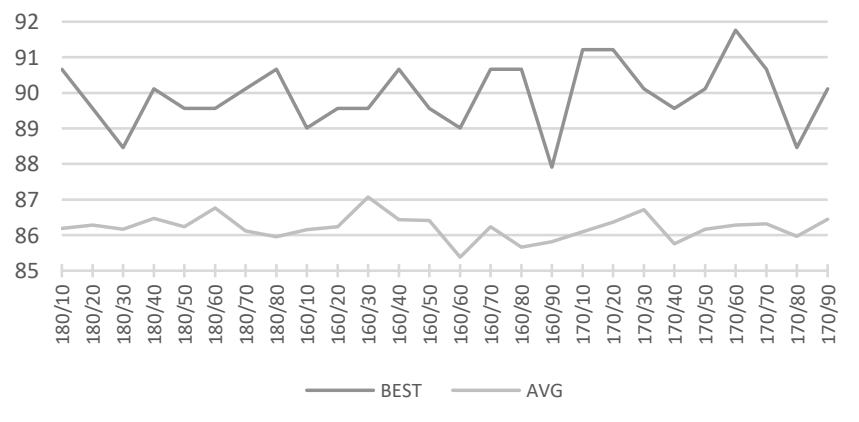

Fig. 6. Results of LSTM with two bi-LSTM layer when incrementing number of hidden units
The single best performing network had 170 units and 60 units in the two layers and achieved an accuracy of $91.76 \%$.

A third layer was added and the same procedure was followed, the number of hidden units was incremented until the average accuracy had peaked. Fig. 7 shows that the performance of the networks, when a third layer is added, never improves upon the two layer setup. A single network reaches 91.76\% when using 170:60:30 hidden units in the respective layers. However, all the tested network configurations do not outperform any of the 2 layer architectures previously tested. Whilst some of the individual networks achieve an accuracy of $89.56 \%$ the average accuracies all drop below $86 \%$ so test up to 50 hidden units were only carried out to confirm the continual drop in classification performance.

Overall there were two network architectures that produced a classification accuracy of $91.67 \%$. This accuracy was achieved when using a two layer LSTM network with 170 units and 60 units in each of the respective layers and the same accuracy was achieved in a three layer architecture when using 170 units, 60 units and 30 units in the respective layers.

Nonetheless, there were other architectures that classified with similar accuracies, e.g. 170 hidden units with either 10 or 20 units in the additional bi-LSTM layers, however, the difference is insignificant at less than $1 \%$.

Further analysis of the confusion matrix, shown in Fig. 8 , shows where the confusion is occurring. A similar pattern across the results show that the main area of confusion is with the movements of the thumb. Thumb extension and flexion

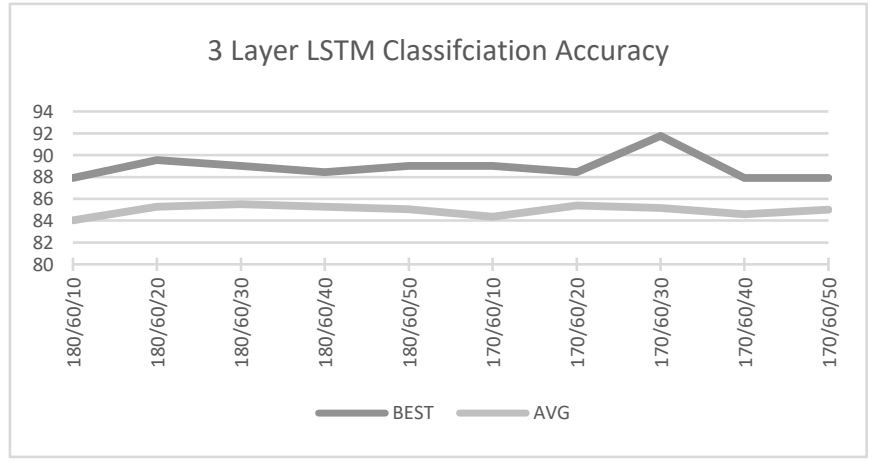

Fig. 7. Results of LSTM with three bi-LSTM layer when incrementing number of hidden units

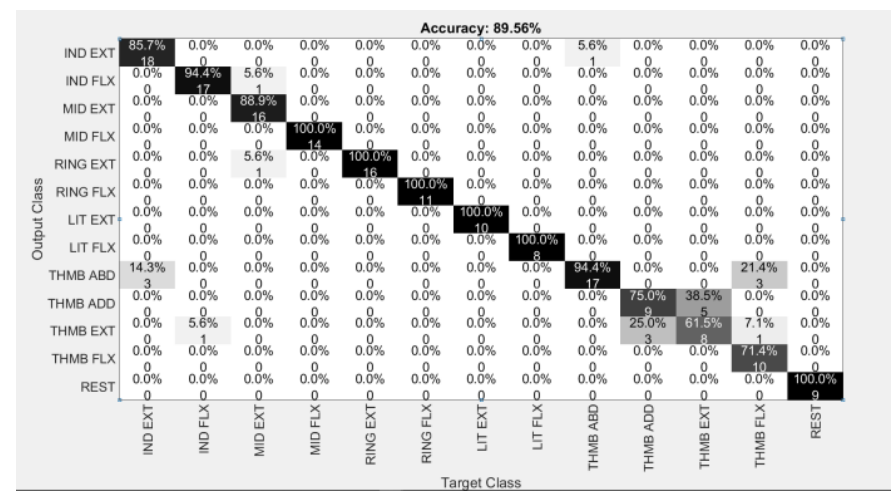

Fig. 8. Confusion Matrix showing breakdown of individual finger movement classification for best performing 2 Layer LSTM 


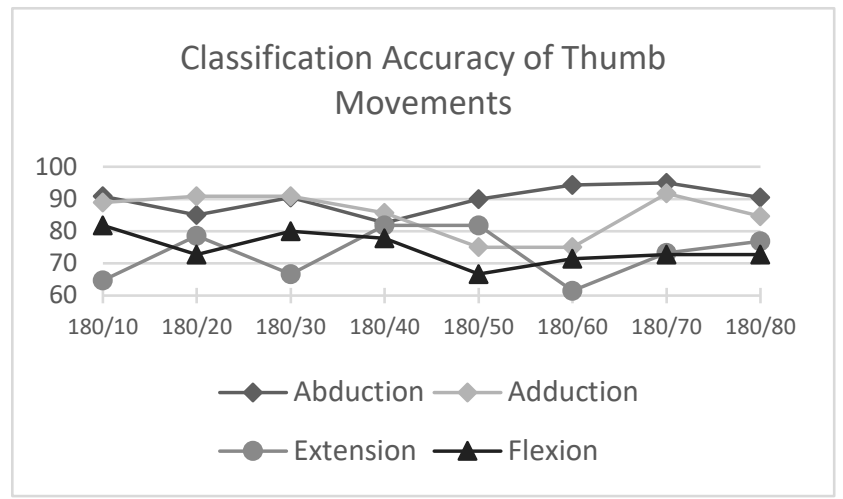

Fig. 9. Accuracy of each thumb movement across each 2 layer LSTM

movements demonstrate to be the most difficult movements to classify with $61.5 \%$ and $71.4 \%$, respectively. These signals were confused with the thumb adduction and abduction movements by the LSTM network. A potential cause of this is due to the fact that the not all the muscles that are responsible for thumb movements are found in the forearm where the signals were being detected from. There also intrinsic muscles found at the base of the thumb that control various aspects of the fine motor controls that the thumb can perform. These confusions of the thumb movements are consistent throughout the networks, Fig. 9 shows the varying levels of classification accuracy achieved when classifying these movements.

The closest comparison that can be made is with [23] where an accuracy of $71.66 \%$ was reported when classifying the same 12 movement signals. This research has improved on that finding with an average accuracy of $86.76 \%$ and some individual networks achieving $90 \%$ accuracy.

\section{CONCLUSION}

This paper has demonstrated that Myo gesture control armband in combination with an LSTM network can be effectively implemented as a method of sEMG signal classification. The results of the experiments carried out show that whilst finger movements can be successfully classified they are much more difficult to classify if compared to classification of gestures that involve larger movements of the hand that involve multiple fingers or movements of the arm and wrist.

Developing a network that can successfully classify sEMG signals will allow further advancements in robotic object manipulation. Using human demonstrators with wearable sEMG devices and in combination with LSTM classifiers can be used not only for dexterous control of anthropomorphic robotic hands but also can be used as a method of informing a robotic grasping system.

To enhance the generality of these networks the addition of data from a range of subjects will be carried out. Further analysis of LSTM network parameters and how they affect the classification accuracy of the trained networks is needed in order to optimize the performance i.e. mini batch size, max epochs, number of hidden units and addition of frequency domain features. With the thumb showing to be most difficult to classify an investigation into networks that classify finger movement's vs thumb movements as well as introduction of additional sEMG collection technology that can focus on the muscles responsible for thumb movements that are found within the hand.

\section{REFERENCES}

[1] R. Hodson, “A gripping problem,” Nature, vol. 557, no. 7704, pp. S23-S25, 2018.

[2] B. Hudgins, P. Parker, and R. N. Scott, "A New Strategy for Multifunction Myoelectric Control," IEEE Trans. Biomed. Eng., vol. 40, no. 1, pp. 82-94, 1993.

[3] M. Zardoshti-Kermani, B. C. Wheeler, K. Badie, and R. M. Hashemi, "EMG Feature Evaluation for Movement Control of Upper Extremity Prostheses," IEEE Trans. Rehabil. Eng., vol. 3, no. 4, pp. 324-333, 1995.

[4] F. Tenore, A. Ramos, A. Fahmy, S. Acharya, R. EtienneCummings, and N. V. Thakor, "Towards the Control of Individual Fingers of a Prosthetic Hand Using Surface EMG Signals," in 2007 29th Annual International Conference of the IEEE Engineering in Medicine and Biology Society, 2007, pp. 6145-6148.

[5] A. Phinyomark, S. Hirunviriya, C. Limsakul, and P. Phukpattaranont, "Evaluation of EMG feature extraction for hand movement recognition based on Euclidean distance and standard deviation," Electr. Eng. Comput. Telecommun. Inf. Technol. (ECTICON), 2010, no. May, pp. 856-860, 2010.

[6] L. J. Hargrove, K. Englehart, and B. Hudgins, "A Comparison of Surface and Intramuscular Myoelectric Signal Classification," IEEE Trans. Biomed. Eng., vol. 54, no. 5, pp. 847-853, May 2007.

[7] F. V. G. Tenore, A. Ramos, A. Fahmy, S. Acharya, R. EtienneCummings, and N. V. Thakor, "Decoding of Individuated Finger Movements Using Surface Electromyography," IEEE Trans. Biomed. Eng., vol. 56, no. 5, pp. 1427-1434, May 2009.

[8] R. M. Stephenson, R. Chai, and D. Eager, "Isometric Finger Pose Recognition with Sparse Channel SpatioTemporal EMG Imaging," Proc. Annu. Int. Conf. IEEE Eng. Med. Biol. Soc. EMBS, vol. 2018July, pp. 5232-5235, 2018.

[9] M. Atzori et al., "Electromyography data for non-invasive naturally-controlled robotic hand prostheses," Sci. Data, vol. 1, pp. 1-13, 2014.

[10] A. Phinyomark, P. Phukpattaranont, and C. Limsakul, "Feature reduction and selection for EMG signal classification," Expert Syst. Appl., vol. 39, no. 8, pp. 7420-7431, Jun. 2012.

[11] M. R. Ahsan, M. I. Ibrahimy, and O. O. Khalifa, "Electromygraphy (EMG) signal based hand gesture recognition using artificial neural network (ANN)," 2011 4th Int. Conf. Mechatronics Integr. Eng. Ind. Soc. Dev. ICOM'11 - Conf. Proc., no. May, pp. 1-6, 2011.

[12] S. Benatti et al., "A Versatile Embedded Platform for EMG Acquisition and Gesture Recognition," IEEE Trans. Biomed. Circuits Syst., vol. 9, no. 5, pp. 620-630, 2015.

[13] A. Phinyomark and E. Scheme, "A feature extraction issue for myoelectric control based on wearable EMG sensors," in 2018 IEEE Sensors Applications Symposium (SAS), 2018, pp. 1-6. 
[14] I. Mendez et al., "Evaluation of the Myo armband for the classification of hand motions," IEEE Int. Conf. Rehabil. Robot., pp. 1211-1214, 2017.

[15] M. Atzori et al., "A Benchmark Database for Myoelectric Movement Classification,” Trans. Neural Syst. Rehabil. Eng., 2013.

[16] K. Nymoen, M. Romarheim, H. Alexander, and R. Jensenius, "MuMYO - Evaluating and Exploring the MYO Armband for Musical Interaction," New Interfaces Music. Expr., p. Paper 179, 2015.

[17] S. Shin, Y. Baek, J. Lee, Y. Eun, and S. H. Son, "Korean sign language recognition using EMG and IMU sensors based on groupdependent NN models," 2017 IEEE Symp. Ser. Comput. Intell. SSCI 2017 - Proc., vol. 2018-Janua, pp. 1-7, 2018.

[18] A. Phinyomark, C. Limsakul, and P. Phukpattaranont, "A Novel Feature Extraction for Robust EMG Pattern Recognition,” J. Med. Eng. Technol., vol. 1, no. 1, pp. 71-80, Dec. 2009.

[19] K. Tatarian, M. S. Couceiro, E. P. Ribeiro, and D. R. Faria, "Stepping-stones to Transhumanism: An EMG-controlled Low-cos Prosthetic Hand for Academia," no. November, pp. 807-812, 2019.

[20] M. E. Benalcazar, A. G. Jaramillo, Jonathan, A. Zea, A. Paez, and V. H. Andaluz, "Hand gesture recognition using machine learning and the Myo armband," in 2017 25th European Signal Processing Conference (EUSIPCO), 2017, vol. 2017-Janua, pp. 1040-1044.

[21] M. E. Benalcazar et al., "Real-time hand gesture recognition using the Myo armband and muscle activity detection," 2017 IEEE 2nd Ecuador Tech. Chapters Meet. ETCM 2017, vol. 2017-Janua, pp. 16, 2018.

[22] S. R. Kurniawan and D. Pamungkas, "MYO Armband sensors and Neural Network Algorithm for Controlling Hand Robot," Proc. 2018 Int. Conf. Appl. Eng. ICAE 2018, pp. 1-6, 2018.

[23] Y. Wu, B. Zheng, and Y. Zhao, "Dynamic Gesture Recognition Based on LSTM-CNN," Proc. 2018 Chinese Autom. Congr. CAC 2018, pp. 2446-2450, 2019.

[24] K. Akhmadeev, E. Rampone, T. Yu, Y. Aoustin, and E. Le Carpentier, "A testing system for a real-time gesture classification using surface EMG," IFAC-PapersOnLine, vol. 50, no. 1, pp. 11498-11503, 2017.
P. Paudyal, J. Lee, A. Banerjee, and S. K. S. Gupta, “A comparison of techniques for sign language alphabet recognition using armband wearables," ACM Trans. Interact. Intell. Syst., vol. 9, no. 2-3, 2019.

R. N. Khushaba, A. Al-Ani, and A. Al-Jumaily, "Orthogonal Fuzzy Neighborhood Discriminant Analysis for Multifunction Myoelectric Hand Control," IEEE Trans. Biomed. Eng., vol. 57, no. 6, pp. 14101419, Jun. 2010

[27] M. Khezri and M. Jahed, "A neuro-fuzzy inference system for sEMG-based identification of hand motion commands," IEEE Trans. Ind. Electron., vol. 58, no. 5, pp. 1952-1960, 2011.

[28] A. D. C. Chan and G. C. Green, "Myoelectric control development toolbox," Proc. 30th Conf. Can. Med. Biol. Eng. Soc., vol. 1, no. September, pp. M0100-1, 2007.

[29] A. H. Al-Timemy, G. Bugmann, J. Escudero, and N. Outram, "Classification of finger movements for the dexterous hand prosthesis control with surface electromyography," IEEE J. Biomed. Heal. Informatics, vol. 17, no. 3, pp. 608-618, 2013.

[30] F. Quivira, T. Koike-Akino, Y. Wang, and D. Erdogmus, "Translating sEMG signals to continuous hand poses using recurrent neural networks," 2018 IEEE EMBS Int. Conf. Biomed. Heal. Informatics, BHI 2018, vol. 2018-Janua, no. March, pp. 166169, 2018.

[31] A. Gijsberts, M. Atzori, C. Castellini, H. Müller, and B. Caputo, "Movement error rate for evaluation of machine learning methods for sEMG-based hand movement classification," IEEE Trans. Neural Syst. Rehabil. Eng., vol. 22, no. 4, pp. 735-744, 2014.

[32] S. Pizzolato, L. Tagliapietra, M. Cognolato, M. Reggiani, H. Müller, and M. Atzori, "Comparison of six electromyography acquisition setups on hand movement classification tasks," PLoS One, vol. 12, no. 10, pp. 1-17, 2017.

[33] S. Hochreiter and J. Urgen Schmidhuber, "Long Shortterm Memory,” Neural Comput., vol. 9, no. 8, p. 17351780, 1997.

[34] T. Cooijmans, N. Ballas, C. Laurent, Ç. Gülçehre, and A. Courville, "Recurrent batch normalization," in 5th International Conference on Learning Representations, ICLR 2017 - Conference Track Proceedings, 2019, no. Section 3, pp. 1-13.

[35] M. Tomaszewski;, "Myo SDK MATLAB MEX Wrapper;” 2019.

[36] M. Dwarampudi and N. V. S. Reddy, "Effects of padding on LSTMs and CNNs," Mar. 2019. 